\title{
BIBLIOGRAFIA DE Y SOBRE CLARICE LISPECTOR
}

POR

EARL F. FITZ

Pennsylvania State University

\section{INTRODUÇÃo}

Pretendo que esta bibliografia seja útil e atual para todos aqueles que queiram conhecer melhor a obra de Clarice Lispector. Todavia, considero que a mesma não é absolutamente completa. Não incluimos, por exemplo, os comentários jornalísticos que são de valor efêmero ou que não expressam nenhuma informação nova sobre a ficção da escritora.

O princípio que orienta esta compilação bibliográfica é a acumulação e organização de todas as primeiras edições dos romances, contos e crônicas publicados pela estimada ficcionista, bem como de todos os estudos críticos importantes acerca de sua obra, incluindo livros, artigos e revistas. Cada referência contém toda a informação que até o presente momento pude investigar. Já examinei pessoalmente a grande maioria das citações que relacionei, mas, em certos casos, os que estão assinalados com asteriscos (*), foram realizados segundo fontes secundárias. Em tais casos, a documentação não é de confiança cabal e, por isso, o leitor é avisado a fazer mais pesquisas para completar a sua exatidão.

Espero que esta modesta contribuição ao entendimento da obra de Clarice Lispector incentive e facilite estudos mais aprofundados e extensivos sobre a sua vida e arte. Se isto acontecer, então considero que esta bibliografia de e sôbre Clarice Lispector terá sido útil e assim ficarei muito compensado. 


\section{ObRas de Clarice Lispector}

Lispector, Clarice. A Bela e a Fera. Rio de Janeiro: Editôra Nova Fronteira, 1979.

- A Agua Viva. Rio de Janeiro: Editôra Artenova, 1973.

- Alguns Contos. Rio de Janeiro: Ministério de Educação e Saúde, 1952.

- The Apple in the Dark. Trans. and with an introd. by Gregory Rabassa. New York: Alfred A. Knopf, 1967.

- Uma Aprendizagem ou o Livro dos Prazeres. Rio de Janeiro: Editôra Sabiá, 1969.

- A Cidade Sitiada. Rio de Janeiro: Editôra Sabiá, 1948.

- De Corpo Inteiro. Rio de Janeiro: Editôra Artenova, 1975.

-. «Divagando Sobre Tolices». Correio do Povo, Vol. VI, ano III, No. 113, 13 junho, 1970, pp. 2-3.

-. «A explicação que não explica». Jornal do Brasil, Rio de Janeiro, 11 de outubro, 1969.

- - "Excerpts from The Foreign Legion». Trans, and with an introd. by Giovanni Pontiero. Review 24 (June) 1979, pp. 37-43.

—. Family Ties. Trans. and with an introd. by Giovanni Pontiero. Austin: University of Texas Press, 1972.

—. Felicidade Clandestina: Contos. Rio de Janeiro: Editôra Sabiá, 1971.

-. A Hora da Estrela. Rio de Janeiro: Livraria José Olympio Editôra, 1977.

—. A Imitação da Rosa. Rio de Janeiro: Editôra Artenova, 1973.

—. Laços de Família. São Paulo: Francisco Alves Editôra, 1960.

- A Legião Estrangeira. Rio de Janeiro: Editôra do Autor, 1964.

-. «Literatura de Vanguardia no Brasil». Movimientos Literarios de Vanguardia en Iberoamérica: Memoria del Undécimo Congreso (sic). Austin: La Universidad de Texas, 1965, pp. 109-116.

—. O Lustre. Rio de Janeiro: Editôra Agir, 1945.

—. A Maçã no Escuro. Rio de Janeiro: Livraria F. Alves, 1961.

-. "Miopia Progressiva». Correio do Povo, Vol. VI, ano III, No. 140, 1 de agosto, 1970 , pp. 2-3.

—. O Mistério do Coelho Pensante. Rio de Janeiro: Editôra José Alvaro, 1967.

-. A Mulher que Matou os Peixes. Rio de Janeiro: Editôra Sabiá, 1968.

-. Onde Estivestes de Noite. Rio de Janeiro: Editôra Artenova, 1974.

- - A Paixão Segundo G. H. Rio de Janeiro: Editôra do Autor, 1964.

- _. Para não Esquecer. São Paulo: Editôra Ática, 1978.

- Perto do Coração Selvagem. Rio de Janeiro: A Noite, 1944.

- - Quase de Verdade. Rio de Janeiro: Editôra Rocco Ltda., 1978.

—. O Retrato de Dorian Grey [Tradução de Clarice Lispector]. Rio de Janeiro: Edições de Ouro, 1974.

- Seleta de Clarice Lispector. Seleção e textomontagem do Professor Renato Cordeiro Gomes, com estudos e notas do Professor Amariles Guimarães Hill. Brasília: Instituto Nacional do Livro/Ministério da Educação e Cultura, 1975. -. «Sofia's Disasters». Trans. by Elizabeth Lowe. Review 24 (June) 1979, pp. 27-33.

- Um Sopro de Vida. Rio de Janeiro: Editôra Nova Fronteira, 1978.

- - A Via Crucis do Corpo. Rio de Janeiro: Editôra Artenova, 1974. 
- - A Vida Intima de Laura. Rio de Janeiro: Livraria José Olympio Editôra, 1974.

- Cisão do Esplendor: Impressões Leves. Rio de Janeiro: Livraria Francisco Alves, 1975.

\section{Traduções}

Perto do Coração Selvagem

Près du Coeur Sauvage (Plon, Paris).

Elizko Divokého Srdce Zivota (Tchecoslováquia).

A Sair: Tradução da Editorial Sudamericana, Buenos Aires.

A Sair: Tradução Espanhola, Barcelona.

O Lustre

A Sair: Tradução, Buenos Aires.

Laços de Familia

Family Ties (University of Texas Press, Estados Unidos).

Die Nachahmung der Rose (Classen Verlag, Alemanha).

A Sair: Buenos Aires.

A Maçã no Escuro

Le bâtisseur des ruines (Gallimard, Paris).

The Apple in the Dark (Knopf, New York).

Der Apfel im Dunkeln (Classen Verlag, Alemanha).

Mistério do Coelho Pensante

A Sair: Ediciones de la Flor, Buenos Aires.

A Paixão Segundo G. $H$.

La Pasión según G. H. (Monte Avila, Caracas).

La Passion selon G. H. (Des Femmes, Paris).

Uma Aprendizagem

A Sair: Editorial Sudamericana, Buenos Aires.

A Sair: Milão, Itália.

"The Smallest Woman in the World». Trans. by Elizabeth Bishop. The Eye of the Heart, New York: Bard/Avon, 1973, pp. 447-454.

«Marmosets», Trans. by Elizabeth Bishop. The Eye of the Heart, New York: Bard/ Avon, 1973, pp. 455-457.

«The Woman Who Killed the Fish». Trans. by Earl E. Fitz. Scheduled to appear in a forthcoming issue of the Latin American Literary Review.

«The Man Who Appeared». Trans. by Alexis Levitin. Latin American Literature Today, Anne Fremantle, ed. New York: New American Library, 1977, pp. 165169.

«Better Than to Burn». Trans. by Alexis Levitin. Latin American Literature Today, 1977, pp. 169-171.

«Temptation». Trans. by Elizabeth Lowe. Inter-Muse, Vol. 1, No. 1, 1976, pp. 91-92.

\section{Livros Sôbre Clarice Lispector}

Borelli, Olga. Clarice Lispector («Esboço para um Possível Retrato»). Rio de Janeiro: Editôra Nova Fronteira, 1981.

Brasil, Assis. Clarice Lispector. Rio de Janeiro: Editôra Organização Simões, 1969. 
Nunes, Benedito. O Mundo de Clarice Lispector. Manaus: Edições Govêrno do Estado do Amazonas, 1966.

- Leitura de Clarice Lispector. São Paulo: Editôra Quíron, 1974.

Pereira, Teresinha Alves. Estudo sôbre Clarice Lispector. Coimbra: Edições Nova Era, 1975.

Sá, Olga de. A Escritura de Clarice Lispector. Petrópolis: Vozes; Lorena: Faculdades Integradas Teresa d'Avila, 1979.

\section{Artigos e ensaios em tôRno de Clarice Lispector}

Abreu, Caio Fernando. «Por Telepatia». Veja, 9 de janeiro 1980, pp. 65-66.

Aderbal, Jurema. Poetas e Romancistas do Nosso Tempo. Recife: Editồra Nordeste, 1953, p. 90.

Anônimo. «A literatura, segundo Clarice» (entrevista). Minas Gerais, Suplemento Literário, 28 setembro 1968, pp. 8-9.

Anônimo. "Clarice parte da intuição». Visão, 8 dezembro 1961, p. 71.

Almeida, Roberto Wagner de. «G. H. e Dardará». Estado de São Paulo, Suplemento Literário, 17 junho 1967, p. 4.

Amora, Antônio Soares. História da Literatura Brasileira, 7. edição. São Paulo: Edição Saraiva, 1955, pp. 163-174.

An Anthology of Brazilian Prose: From the Beginnings to the Present Day. Eds. R. L. Scott-Buccleuch and Mário Teles de Oliveira. São Paulo: Editôra Ática, 1971, pp. 328; pp. 524-530.

Araújo, Laís Corrêa de. «Moldura e mágica da palavra». Estado de São Paulo, Suplemento Literário, 6 setembro 1969, p. 1.

—. «'A Paixão’ é a linguagem». Minas Gerais, Suplemento Literário, 28 setembro 1968, pp. 10-11.

Assumpção, Sebastião G. "A Paixão segundo G. H.». Diário de Notícias, Suplemenio Literário, Rio de Janeiro, 6 fevereiro $1966^{*}$.

Ayala, Walmir. «'A Paixão segundo G. H.' Um Romance de doação». Jornal do Comércio, Rio de Janeiro, 1 dezembro $1964^{*}$.

Azevedo Filho, Lodegário A. de. «Clarice Lispector e a Visão do Invisível». Minas Gerais, Suplemento Literário, 18 fevereiro, 1978, p. 3.

Bairão, Reynaldo. «Nada existe que escape a transfiguração». Estado de São Paulo, Suplemento Literário, 2 agosto 1969, p. 6.

- _. "Novos apontamentos para um estudo sôbre Clarice Lispector». Estado de São Paulo, Suplemento Literário, 23 agosto 1969, p. 4.

Barbosa, Rolmes. «Mapa dos caminhos sem saída». Estado de São Paulo, Suplemento Literário, 30 junho 1974, p. 2.

The Borzoi Anthology of Latin American Literature, Vol. II. Eds. Monegal e Colchie. New York: A. Knopf, 1977, pp. 779-792.

Brasil, Assis. «Clarice Lispector e a ficção moderna». Jornal de Letras, 1968, 218, p. 3; 219, p. 3; 220, p. 3 .

-. «Laços de Família». Jornal do Brasil, Rio de Janeiro, 24 setembro, 1960 *.

- «O mundo subjetivo de Clarice Lispector». Jornal do Brasil, Rio de Janeiro, novembro $6,13,19$ e dezembro $3,10,1960$ *.

* Indica que não se sabe a paginação exata. 
-... «Paixão e queda de G. H.». Jornal de Letras, Rio de Janeiro, fevereiro/março 1965 *.

- (Outros artigos de A. Brasil sobre Clarice Lispector). Jornal de Letras, Rio de Janeiro, ano XX, Números 210, 219, 220, 221, 222, $225 *$.

Brasil: Prosa e Poesia. Antologia. Ed. Silviano Santiago. New York: Las Américas Publishing Co., 1969, pp. 60-70.

Bruno, Haroldo. "Água Viva, um solilóquio de Clarice Lispector sobre o Ser». Estado de São Paulo, Suplemento Literário, 3 fevereiro 1974, p. 1.

- «Hibridismo de gêneros». Estado de São Paulo, Suplemento Literário, 15 dezembro 1974 , p. 1.

-. «Presença Renovadora de Clarice Lispector». Minas Gerais, Suplemento Literário, 14 julho, 1979, pp. 6-7.

Bryan, C. D. B. (A review of). The Apple in the Dark. New York Times Book Review, 3 September 1967, pp. 22-23.

Burlá, Eliezer. «Perto do Coração Selvagem». O Jornal, Rio de Janeiro, 31 março 1955 *.

Cândido, Antônio. Brigada Ligeira. São Paulo: Livraria Martins, 1945.

—. Vários Escritos. São Paulo: Editôra Duas Cidades, 1970, pp. 123-131.

-. "No Raiar de Clarice Lispector». Vários Escritos, São Paulo: Livraria Duas Cidades, 1970, pp. 125-131.

Cândido, Antônio and Castello, J. Alderado. Presença de Literatura Brasileira, III, Modernismo, 3 série. São Paulo: Difusão.

Castellanos, Rosario. «Clarice Lispector: la memoria ancestral». Mujer que sabe latín (México: Sep-Setentas, 1973), p. 129.

Castro, Sílvio. A Revolução da Palavra (Petrópolis: Vozes, 1976), pp. 263-267.

Chagas, Wilson. «Clarice Lispector», (em) Provincia de São Pedro, No. 15, 1951, pp. 90-92.

Cixous, Helene. «L'approche de Clarice Lispector: Se laisser lire (por) Clarice Lispector - A paixão segundo G. H.». Poetique, Vol. 40, Nov. 1979, pp. 408-419.

Coelho, Nelson. "Clarice». Jornal do Brasil, Rio de Janeiro, 20 agosto 1960 *.

O Conto Feminino: Panorama do Conto Brasileiro, Vol. 10. Ed. R. Magalhães, Junior. Rio de Janeiro: Editôra Civilização Brasileira, 1959.

Cook, Bruce. "Women in the Web». Review 73 (Spring), 1973, pp. 65-66.

Coutinho, Afrânio. Antologia Brasileira de Literatura, Vol. 1 (Romance e Conto). Rio de Janeiro: Editôra Distribuidora de Livro Escolares Ltda., 1965.

- An Introduction to Literature in Brazil. Trans. by. Gregory Rabassa. New York: Columbia University Press, 1969, p. 249.

Crawford, William Rex. «Algunos libros brasileños recientes». Revista lberoamericana, VIII, 1944, pp. 335-341.

David, Carlos. «Em tôrno de Clarice Lispector». Diário Carioca, Río de Janeiro, 3 maio $1953 *$ :

Dicionário Crítico do Moderno Romance Brasileiro. Ed. Pedro Américo Maia; et al., Belo Horizonte: Grupo Gente Nova, 1970.

Dicionário de Literatura Portuguesa e Brasileira. Ed. Celso Pedro Luft. Porto Alegre: Editôra Globo, 1966, pp. 152-154.

Encyclopedia of World Literature in the Twentieth Century, Vol. 4. Eds. Frederick Ungar and Lina Mainiero. New York: Frederick Ungar Publishing Co., 1975, pp. 220-223.

Escorel, Lauro. «Crítica Literária». A Manhã; Rio de Janeiro, 20 outubro 1944 *. 
«Prêmio da Fundação Graça Aranha de 1943». A Manhã, Rio de Janeiro, 29 outubro 1944 *.

Ferreira, Jurandir. «Do estilo do homem de Deus». Estado de São Paulo, Suplemento Literário, 3 fevereiro 1962 *.

-. «Itinerário da Romancista». Diário Carioca, Rio de Janeiro, 25 junho 1956 *. Filho, Adonias. Modernos Ficcionistas Brasileiros. 2 série. Rio de Janeiro: Edições Tempo Brasileiro, 1965, pp. 81-83.

Filho, Azevedo. Síntese Crítica da Literatura Brasileira. Rio de Janeiro: Edições Gernasa, 1971.

Filho, O. C. Lousada. «La Ficción en Crisis: Problemas del Autor Brasileño». Nueva Narrativa Hispanoamericana, Vol. II, enero 1973, núm. 1, pp. 9-19.

Filho, Remy G.: «Clarice Lispector: 'E não sou um monstro sagrado'». Revista das Letras, No. 41, 1971, pp. 112-115.

Finamor, Jurema. «Clarice Lispector». Jornal de Letras, Rio de Janeiro, ano XII, No. $143 \%$.

Fitz, Earl E. «Clarice Lispector and the Lyrical Novel: A Re-examination of A Maça no Escuro». Luso-Brasilian Review, Vol. 14, No. 2, Winter 1977, pp. 153-160.

- «Freedom and Self-Realization: Feminist Characterization in the Fiction of Clarice Lispector». Modern Language Studies, Vol. 10, No. 3, 1980, pp. 51-61.

- - «Clarice Lispector: The Nature and Form of the Lyrical Novel». Dissertation Abstracts International: 37: 7119 A (C. U. N. Y., 1977).

- - "The Leitmotif of Darkness in Seven Novels by Clarice Lispector». Chasqui: Revista de Literatura Latinoamericana, Vol. VII, No. 2, febrero 1978, pp. 18-28.

- . "The Rise of the New Novel in Latin America: A Lyrical Aesthetic». Intermuse, Vol. 2, 1919, pp. 17-27.

- «Point of View in Clarice Lispector's A Hora da Estrela». Luso-Brazilian Review, Winter 1982, Vol. 19, No. 2, pp. 195-208.

Fonta, Sérgio. «O Papo: Clarice Lispector». Jornal de Letras, No. 259, Cad. 1, 1972 , p. 5.

Garcia, Frederick C. H. «Os Livros Infantis de Clarice Lispector». Minas Gerais, Suplemento Literário, 10 fevereiro 1979, pp. 4-5.

- «Uma Visão de Clarice Lispector». Minas Gerais, Suplemento Literário, Vol. 26, No. 721,3 julho 1980, p. 3.

Gilio, María Ester. Interview with Clarice Lispector in Crisis (Buenos Aires), No. 39 (1976), p. 42.

Goldman, Richard Franko. "The Apple in the Dark». Saturday Review, New York, 19 August 1967, pp. 33, 48.

Hécker, Paulo Filho. "Uma mística em tempo de Deus morto». Alguma Crítica. Porto Alegre, 1952, pp. 94-95.

- Ibid. Diário. Porto Alegre, 1949, pp. 251-260.

—. Ibid. Estado de São Paulo, Suplemento Literário, 4 outubro 1969, p. 6.

-. "Itinerário de romancista». Diário Carioca, Rio de Janeiro, 25 junho 1956 *.

Helena, Lúcia. «Aprendizado de Clarice Lispector». Littera: Revista para Professores de Português e de Literaturas de Lingua Portuguesa, 13, 1975, pp. 99-104.

- - «O discurso do silêncio: A Narrativa dinâmica de Clarice Lispector». O Estado de São Paulo, Suplemento Literário, 11 agosto 1974, p. 3.

Herman, Rita. «Existence in Laços de Familia». Luso-Brazilian Review, Vol. IV, No. 1, Spring (June) 1967, pp. 69-74.

Hill, Amariles Guimarões. «O Sistema Original de Clarice Lispector». Tempo Brasileiro: Revista de Cultura, Vol. 48, 1977, pp. 52-71. 
Howlett, Jacques. «Pour que L'Horreur Devienne Lumière». Quinzaine Littéraire, No. 293, 1-15, janvier 1979, pp. 11-12 (on La Passion selon G. H.).

Jafa, Van. «Coração Selvagem de Clarice». Correio da Amanhã, Rio de Janeiro, 10 dezembro 1965 *.

Jordão, Yolanda. «Sobre Clarice Lispector». Minas Gerais, Suplemento Literário, 11 março 1978, p. 7.

Jornal de Critica (Anónimo). Rio de Janeiro: Livraria José Olympio Editôra, 1946, Capítulo IX*.

Jornal de Crítica (Anónimo). Rio de Janeiro: Livraria José Olympio Editôra, 5 série, 1947, pp. 152-155.

Jornal de Crítica (Anónimo). Rio de Janeirọ: Livraria José Olympio Editôra, 4 série, 1949, pp. 108-114.

José, Elias. «Anotações sobre Água Viva». Minas Gerais, Suplemento Literário, 26 outubro, p. 2; 2 novembro 1974, pp. 4-5; 9 novembro 1974, pp. 8-9.

Jozef, Bella. «Clarice Lispector: La transgresión como acto de libertad». Revista Iberoamericana, Vol. XLIII, números 98-99, enero-junio 1977, pp. 225-231.

. "Chronology: Clarice Lispector». Trans. by Elizabeth Lowe. Review 24 (June) 1979, pp. 24-26.

- «Le Fantastique dans la littérature hispano-américaine contemporaine». Cahiers du Monde Hispanique et Luso-Brésilien, Vol. 29 (1977), pp. 7-24.

Kerr, Yllen. «Clarice responde...». Jornal do Brasil, 8 setembro 1963 *.

Lauria, Márcio José. «Clarice Lispector: Uma Cosmovisão». Minas Gerais, Suple mento Literário, 17 julho, p. 6; 24 julho, pp. 8-9.

Leite, Ascendino. As Durações: Passado Indefinido, Os Dias Duvidosos, O Lucro de Deus. Belo Horizonte: Editôra Itatiatia, 1966, p. 430.

Lima, Luíz Costa. Lira e Antilira. Rio de Janeiro: Editôra Civilização Brasileira, 1968.

- A Literatura no Brasil, Vol. V. Ed. Afrânio Coutinho. São Paulo: Editôra Sul Americano, 1970, pp. 449-472.

- «A Mística ao Revés de G. H.». Por Que Literatura. Petrópolis: Editôra Vozes, 1966, pp. 92-105.

Lindstrom, Naomi. «Clarice Lispector: Articulating Women's Experience». Chasqui, Vol. 8, No. 1 (1978), pp. 43-52.

_- «A Discourse Analysis of 'Preciosidade" by Clarice Lispector». Luso-Brazilian Review, Vol. 19, No. 2 (Winter) 1982, pp. 187-94.

Linhares, T. «O Novo Romance Brasileiro-VII». Estado de São Paulo, Suplemento Literário, No. 670 *.

-. "Uma Cura de Alma». Estado de São Paulo, 19 novembro 1960 ".

- «Romances Femininos». Estado de São Paulo, 18 novembro 1961 *.

Lins, Álvaro. «A experiéncia incompleta». Os Martos de Sobrecasaca. Rio de Janeiro: Editôra Civilização Brasileira, 1963, pp. 186-193.

- "Perto de Coração Selvagem». Dicionário Crítico do Moderno Romance Brasileiro, Vol. 2, ed. Pedro Maia. Belo Horizonte: Grupo Gente Nova, 1970.

- O Relogio e o Quadrante. Rio de Janeiro: Editôra Civilização Brasileira, 1964. Lins, Osman. «O tempo em 'Feliz Aniversário'». Colóquio/Letras 19, 1974, pp. 16-22. Lispector, Clarice. «Temptation». Trans. by Elizabeth Lowe. Inter Muse, Vol. 1, No. 1, 1976, pp. 91-92.

Lorenz, Günter W. Die Zeitgenössische Literatur in Latinamerika. Tübingen: Horst Erdman Verlag, 1971. 
Lowe, Elizabeth Anne Schlomann. The City in Brazilian Literature. Rutherford: Farleigh-Dickinson University Press, 1982.

- - The Temple and the Tomb: The Urban Tradition in Brazilian Literature and the City in the Contemporary Brazilian Narrative». Dissertation Abstracts International 38: 2826 A (C. U.N. Y., 1977).

- - «The Passion According to C. L.» (Elizabeth Lowe interviews Clarice Lispector). Review 24 (June) 1979, pp. 34-37.

Lucas, Fábio. «Aspectos de la Ficción Brasileña Contemporánea». Nueva Narrativa Hispanoamericana, Vol. III, Núm. 1, enero 1973, pp. 113-123.

- «Contemporary Brazilian Fiction (sic): Guimarães Rosa and Clarice Lispector». [F. 65], 1977, pp. 60-66.

—_. Compromisso Literário. Rio de Janeiro: Livraria São José, 1964.

-. (Em) Contemporary Latin American Literature. Eds. Harvey L. Johnson and Phillip B. Taylor. Houston: University of Houston Press, 1973, pp. 64-66.

- _. «A fome não saturada». Correio da Manhã, Rio de Janeiro, 11 novembro $1961 *$.

- Fronteiras Imaginarias. Rio de Janeiro: Livraria Editôra Cátedra, 1971.

-... "Guimarães Rosa e Clarice Lispector: Mito e Ideologia». Minas Gerais, Suplemento Literário, 26 agosto 1972, pp. 4-5.

- Horizontes da Crítica. Belo Horizonte: Editôra M. P., 1965.

- - «Por que escrever?». Coloquio, Vol. 47, 1979, pp. 74-78.

- Temas Literárias e Juizos Críticos. Belo Horizonte: Editôra Tendência, 1963, p. 153.

Lyotard, J. F. A Fenomenologia. São Paulo: Difusão Europeia do Livro, 1967.

Lyra, Pedro. «O Movimento como a Verdade do Real (Num Romance de Clarice Lispector)». Revista de Letras (Ceará), Vol. 1 (1978), pp. 114-123.

Machado, Âlvaro Manuel. «Na Morte de Clarice Lispector». Coloquio, Vol. 41 (1978), pp. 66-67.

Martins, Heitor. «Fracasso e triunfo de Clarice Lispector». Estado do São Paulo, Suplemento Literário, 15 agosto 1970, p. 6.

- Oswald de Andrade o Outros. São Paulo: Conselho Estadual de Cultura, 1974.

Martins, Teresinha A. P. «Julio Cortázar, Clarice Lispector e a Nova Narrativa Latino-americana». Dissertation Abstracts International 32: 3958 A (N. M., 1972).

Martins, Wilson. «Uma Voz». Estado de São Paulo, Suplemento Literário, 26 novembro 1960, p. 1.

- «O Romance Brasileiro Contemporâneo». Inti, No. III, abril 1976, pp. 27-36.

Mello e Sousa, Gilda de. «O Lustre». O Estado de São Paulo, 14 de julho 1946.

Merquior, José Guilherme. A Astúcia da Mimese. Rio de Janeiro: Livraria José Olympio Editôra, 1972.

- A Razão do Poema. São Paulo: Civilização Brasileira, 1965.

Milliet, Sérgio. «Com veleidade a crônica». Diário Crítico, Vol, 2. São Paulo: Editôra Brasiliense, 1945, pp. 27-32.

—. Diário Crítico, Vol. 4, 1945, pp. 40-44.

—. Diário Crítico, Vol. 7, 1953, pp. 33-44.

—. Diário Crítico, Vol. 8, 1955, pp. 235-237.

-. "A Cidade Sitiada». Letras e Artes, 11 setembro 1949, p. 7.

Modern Brazilian Shori Stories. Trans. and with an introd. by William L. Grossman. Berkeley: University of California Press, 1967. 
Modern Latin American Literature, Vol. I. Eds. David W. Foster and Virginia R. Foster. New York: Frederick Ungar Publishing Co., 1975, pp. 484-491.

Moisés, Massaud. "Clarice Lispector Contista». Temas Brasileiros. São Paulo: Conselho Estadual de Cultura, 1964, pp. 119-124.

- - «Clarice Lispector: Ficção e Cosmovisão». Estado de São Paulo, Suplemento Literário, 26 setembro 1970, p. 1.

- «Clarice Lispector: Fiction and Cosmic Vision». Trans. by Sara M. McCabe. Studies in Short Fiction, Vol. VIII, Number 1, Winter 1971, pp. 268-281.

- A Literatura Brasileira Através dos Textos. São Paulo: Editôra Cultrix, 1971, pp. 507.511.

- Temas Brasileiros. São Paulo: Conselho Estadual de Cultura, 1964, pp. 119 124.

Monegal; Emir Rodríguez. Et Boom de la Novela Latinoamericana. Caracas: Editorial Tiempo Nuevo, 1972, pp. 27 y 93.

-. «The Contemporary Brazilian Novel». Fiction in Several Languages. Ed. Henri Peyre. Boston: Houghton Mifflin Co., Daedalus Library, Vol. 9, 1968, pp. 1-18.

Mourão-Ferreira, David. "Clarice Lispector»: Motim Literário. Lisboa: Editorial Verbo, 1961, pp. 135-139.

Moutinho; Nogueira: «O Livro dos Prazeres». Fôlha de São Paulo, 31 agosto 1969 *.

- _. "O mundo de Clarice Lispector». Estado de São Paulo, Suplemento Literário, No. $483 *$.

—. A Procura do Número. São Paulo: Conselho de Cultura, 1967.

Naudet, Haroldo. «O tema da liberdade nas narrativas de Clarice Lispector». Minas Gerais, Suplemento Literário, 29 abril 1972, pp. 6-7.

Nunes, Benedito. «O mundo imaginário de Clarice Lispector». O Dorso do Tigre. São Paulo: Editôra Perspectiva, 1969, pp. 93-139.

- - "A náusea em Clarice Lispector». Estado de São Paulo, Suplementó Literîio, 24 julho $1965^{\circ}$.

- - «A Paixão Segundo G. H.». Estado de São Paulo, Suplemento Literário, 4 setembro $1965 *$

Nunes, Maria Luisa. «Narrative Modes in Clarice Lispector's Lacos de Familia: The Rendering of Consciousness». Luso-Brazilian Review, Vol. 14, No. 2, Winter 1977, pp. 174-184.

Olinto, Antonio."A Verdade da Ficção. Rio de Janeiro: Companhia Brasileira de Artes Gráficas, 1966, pp. 213-216.

Oliveira, Marly. "A Cidade Sitiada». Jornal do Comércio. Rio de Janeiro, 17 julho 1966 *.

- «Crítica da crítica». Jornal do Brasil, Rio de Janeiro, novembro 1961 *.

- "- «nterpretação da obra de Clarice Lispector». Jornal do Comércio, Rio de Janeiro, 9 janeiro 1966 *.

- "A Maçã no Escuro». Jornal do Comércio, Rio de Janeiro, 24 julho 1963 ".

—. "A Paixão Segundo G. H.». Correio da Manhã, Rio de Janeiro, 13 março $1965 \%$

—. "A Paixão Segundo G. H.». Jornal do Comércio, Rio de Janciro, 31 jutho $1966 *$.

- «Sobre Clarice Lispector». Correio da Manhã, Rio de Janeiro, 28 julho 1963 *

Paes, José Paulo. «Clarice Lispector», Pequeno Dicionário de Literatura Brasileira. São Paulo: Editôra Cultrix, 1967.

Pacheco, Armando Correia. «Brazilian Literature: Native and Foreign Influences». Odyssey Review, December 1961, I, pp. 114-124. 
Patai, Daphne. «Clarice Lispector and the Clamor of the Ineffable». Kentucky Romance Quarterly, Vol. 27, 1980, pp. 133-149.

Pequeno Dicionário de Litérátura Brasileira. Eds. José Paulo Paes e Massaud Moisés. São Paulo: Editôra Cultrix, 1967.

Pereira, Edgard. «Wander e Clarice». Minas Gerais, Suplemento Literário, 16 agosto 1975 , p. 2 (MGSL 11 outubro, p. 2, contains Clarice's reply).

Pereira, Teresinha Alves. «Clarice Lispector: Anotações de Leitura». Minas Gerais, Suplemento Literário, 16 outubro 1971, p. 8.

- "Coincidencia de la técnica narrativa de Julio Cortázar y Clarice Lispector». Nueva Narrativa Hispanoamericana, Vol. III, enero 1973, núm. 1, pp. 103-111.

- «Os contos parábolas de Clarice Lispector». Minas Gerais, Suplemento Literário, 14 maio 1977 , p. 10.

- - «Los nuevos novelistas brasileños (A propósito de Clarice Lispector)». Revista de Cultura Brasileña, 35, 1973, pp. 109-115.

- - «Recursos estilísticos da Nova Narrativa Brasileira». Jornal de Letras 287, Cad. 1, 1974, p. 6.

- «Releitura e Re-explicação de A Maçã no Escuro». Minas Gerais, Suplemento Literário, 26 abril 1975, pp. 10-11.

-. "Una comparación inesperada: Rayuela y A Maçã no Escuro». Revista Mexicana de Cultura - El Nacional, México City, 4 de agosto 1975, p. 3.

-. "Rayuela e A Maçã no Escuro: duas montagens de realismo mágico» (Reprint of a paper given August 27, 1973, at the XVI Congreso del Instituto Internacional de Literatura, Whitewater, Wisconsin) [F. 106)], pp. 361-366.

- «O realismo mágico nas obras de Cortázar e de Clarice Lispector». Diário de Brasilia, 27 dezembro 1973, pp. 4-6.

Perez, Renard. Escritores Brasileiros Contemporâneos, 2 série. Rio de Janeiro: Editôra Civilização Brasileira, 1964, pp. 69-80.

- «Escritores Brasileiros Contemporâneos». Diário de Notícias, 14 outubro 1926 (sic).

Pessanha, J. A. «Itinerário da Paixão». Cadernos Brasileiros, Rio de Janeiro, ano VII, núm. 29, maio-junho 1965, pp. 63-76.

Pólvora, Hélio. «Clarice Lispector». Revista de Cultura Brasileña 40, 1975, pp. 11-15.

- Graciliano, Machado, Drummond e Outros. Rio de Janeiro: Editôra Alves, 1975.

-. A Força da Ficção. Petrópolis: Editôra Vozes, 1971.

Pontiero, Giovanni, tr. and introd. «Excerpts from the Chronicles of The Foreign Legion». Review, Vol. 24, 1980, pp. 37-43.

- "The Drama of Existence in Laços de Familia». Studies in Short Fiction, Vol. VIII, No. 1, Winter 1977, pp. 256-267.

- - «xxerpts from the Chronicles of The Foreign Legion», with an introd. by Giovanni Pontiero. Review 24 (June) 1979, pp. 37-43.

Portal, Marta. «Una Novedad Editorial Española: La Narrativa Brasileña». Revista de Cultura Brasileña, No. 49, julio 1979, pp. 90-100.

Portella, Eduardo. Dimensões II: Critica Literăria. Rio de Janeiro: Livraria Agir Editôra, 1959.

- "A forma expressional de Clarice Lispector». Jornal do Comércio, Rio de Janeiro, 25 setembro e 9 outubro 1960 \%.

- «O Grito do Silêncio». Tempo Brasileiro: Revista de Cultura, Vol. 51, 1977, pp. 8-10. (Reprint of introduction to Lispector's A Hora da Estrela.) 
-. «O livro aberto de Clarice Lispector». Jornal do Brasil, Rio de Janeiro, 12 julho 1969 *.

Portella, Eduardo, et al. Literatura Brasileira: Vertentes. Rio de Janeiro: Tempo Brasileiro, 1977.

Quiñónez-Gauggel, María Cristina. «El personaje femenino existencial en las novelas de Clarice Lispector y Julio Cortázar». Dissertation Abstracts International, 40: 6300 A, 1980.

Rabassa, Gregory. Critical introduction to The Apple in the Dark (English translation). New York: Knopf, 1967.

- - "La Nueva Narrativa en el Brasil». Nueva Narrativa Hispanoamericana, Vol. II, enero 1972, núm. 1, pp. 145-148.

Reis, Fernando G. «Quem tem mêdo de Clarice Lispector». Revista Civilização Brasileira, No. 17, janeiro e fevereiro (17) 1968, pp. 225-234.

Reis, Roberto. «Epifania em São Cristóvão». Minas Gerais, Suplemento Literário, 8 outubro 1977, pp. 6-7.

Riedel, Dirce Cortes. "O enunciado de uma Aprendizagem». Jornal do Brasil, Suplemento do Livro, Rio de Janeiro *.

Rocha, Diva Vasconcelos. "Laços de Familia ou a entunciação do humor». Minas Gerais, Suplemento Literário, 25 maio 1974, p. 3.

—. «Paixão e Morte do Narrador segundo o Narrador». Minas Gerais, Suplemento Literário, 22 Nov., p. 2; 29 Nov., p. 4, 1980.

Rowet, Samuel. "A Hora da Estrela ou as Frutas da Frota, ou um Ensaio de Crítica Literária Policial». Minas Gerais, Suplemento Literário, 11 março 1979, pp. 8-9.

Sant'Anna, Affonso Romano de. Análise estrutural de Romances Brasileiros. Petrópolis: Editôra Vozes, 1974.

- «Clarice Lispector: a linguagem I». Estado de São Paulo, Suplemento Literário, 2 fevereiro, 1962, p. 2.

- - "Laços de Família e Legião Estrangeira». Análise Estrutural de Romances Brasileiros, Petrópolis: Editôra Vozes, 1973, pp. 180-212.

- - «Linguagem: Clarice e Morávia». Estado de São Paulo, Suplemento Literário, 31 agosto, 7 setembro $1963^{*}$.

Sant'Anna, Affonso Romano and Marina Colasanti. «Dezembro sem Clarice». Escrita, Vol. 27, pp. 20-24 (entrevista).

Schwartz, Roberto. A Sereia e o Desconfiado. Rio de Janeiro: Civilização Brasileira, 1965.

Seniff, Dennis. "Self-Doubt in Clarice's Laços de Família». Luso-Brazilian Review, Vol. 14, No. 2, Winter 1977, pp. 199-208.

Severino, Alexandrino E. «Major Trends in the Development of the Brazilian Short Story». Studies in Short Fiction, Vol. VIII, No. 1, Winter 1971, pp. 199-208.

Silveira, Alcântara. "Clarice e o Romance». Diário de Notícias, Salvador, 14 setembro $1962 *$.

Silverman, Malcolm. Moderna Ficção Brasileira (tradução de João Guilherme Linke), Brasília: Civilização Brasileira, 1978, pp. 70-84.

Simões, João Gaspar. «Clarice Lispector existencialista ou suprarrealista?». A Manhã, Suplemento de Letras e Artes, Rio de Janeiro, 1 outubro $1950 *$.

Sodré, Nelson Werneck. «Prosa brasileira em 1964». Revista Civilização Brasileira, Rio de Janeiro, março 1965, pp. 54-61. 
Szklo, Gilda Salem, «O conto 'O Búfalo' de Laços de Familia e a Questão da Escrita em Clarice Lispector». Minas Gerais, Suplemento Literário, 31 março 1979, p. 6.

Veríssimo, Erico. «O Escritor Brasileiro em Nossos Dias». Luso-Brazilian Review, June 1964, Yol. 1, pp: 81-86.

World Literature since 1945: Critical Surveys of the Contemporary Literature of Europe and the Americas. Eds. Ivar Ivask and Gerd von Wilbert. New York: Frederick Ungar Publishing Co., 1973.

Zagury, Elaine. A palavra e os ecos. Petrópolis: Editôra Vozes, 1970.

-.. "O que diz Clarice de Clarice» (um entrevista com Clarice Lispector). Cadernos Brasileiros, No. 50, novembro/dezembro 1968, pp. 60-79. 